\title{
Decision-Making, Cognitive Distortions and Alcohol Use in Adolescent Problem and Non- problem Gamblers: An Experimental Study
}

ARTICLE in JOURNAL OF GAMBLING STUDIES · FEBRUARY 2016

Impact Factor: 2.29 · DOI: 10.1007/s10899-016-9597-7

READS

31

4 AUTHORS:

Maria Ciccarelli

Second University of Naples

3 PUBLICATIONS 0 CITATIONS

SEE PROFILE

Giovanna Nigro

Second University of Naples 35 PUBLICATIONS 159 CITATIONS

SEE PROFILE
Mark D Griffiths

Nottingham Trent University

754 PUBLICATIONS 15,054 CITATIONS

SEE PROFILE

Marina Cosenza

4. Second University of Naples

17 PUBLICATIONS 33 CITATIONS

SEE PROFILE 
Decision-making, cognitive distortions and alcohol use in adolescent problem and non-problem gamblers: An experimental study

Maria Ciccarelli ${ }^{1}$, Mark D. Griffiths ${ }^{2}$, Giovanna Nigro ${ }^{1}$, Marina Cosenza ${ }^{1}$

1 Department of Psychology, Second University of Naples

2 Department of Psychology, Nottingham Trent University

Corresponding author: Maria Ciccarelli, viale Ellittico, 31, 81100 Caserta, Italy;

e-mail: maria.ciccarelli@unina2.it

\begin{abstract}
In the psychological literature, many studies have investigated the neuropsychological and behavioral changes that occur developmentally during adolescence. These studies have consistently observed a deficit in the decision-making ability of children and adolescents. This deficit has been ascribed to incomplete brain development. The same deficit has also been observed in adult problem and pathological gamblers. However, to date, no study has examined decision-making in adolescents with and without gambling problems. Furthermore, no study has ever examined associations between problem gambling, decision-making, cognitive distortions and alcohol use in youth. To address these issues, 104 male adolescents participated in this study. They were equally divided in two groups, problem gamblers and non-problem gamblers, based on South Oaks Gambling Screen Revised for Adolescents scores. All participants performed the Iowa Gambling Task and completed the Gambling Related Cognitions Scale and the Alcohol Use Disorders Identification Test. Adolescent problem gamblers displayed impaired decision-making, reported high cognitive distortions, and had more problematic alcohol use compared to non-problem gamblers. Strong correlations between problem gambling, alcohol use, and
\end{abstract}


cognitive distortions were observed. Decision-making correlated with interpretative bias. This study demonstrated that adolescent problem gamblers appear to have the same psychological profile as adult problem gamblers and that gambling involvement can negatively impact on decisionmaking ability that, in adolescence, is still developing. The correlations between interpretative bias and decision-making suggested that the beliefs in the ability to influence gambling outcomes may facilitate decisionmaking impairment.

Keywords: Decision-making; Cognitive distortions; Alcohol use; Problem gambling; Adolescence 


\section{Introduction}

Human quality of life depends on the ability to make good choices. This ability is particularly critical during child and adolescent development. Adolescents are required to make important decisions that affect the future, such as which subjects to study educationally and their choice of friends. However, during this period, decisional ability has been shown not to be optimal. Over the last few decades, many researchers have become interested in understanding the acquisition of the decision-making ability across the life span.

The most frequently used assessment of decision-making is the Iowa Gambling Task (IGT; Bechara et al., 1994). The IGT requires participants to select one card at time from four decks of cards, each of which gives varying amounts of pay-offs and losses. Two decks are disadvantageous in the long-term because they lead to net losses after repeated choices, while the other two are advantageous because they result in net wins. After sampling from all decks, research has demonstrated that healthy individuals gradually adopt the strategy of selecting cards from the advantageous decks, avoiding those that turn out disadvantageous in the long-term (Bechara et al., 1994).

Many studies on child and adolescent samples have demonstrated that the performance on the IGT improves with age (Cassotti et al., 2011; Cauffman et al., 2010; Crone et al., 2005). In one study (Hooper et al., 2004), three different age cohorts were compared (9-10 years, 11-13 years, 14-17 years). Adolescents aged 14- to 17-years old were found to make more advantageous choices when performing the IGT and selected choices to the advantageous decks earlier than the other two groups. This study also found that age was one of the strong predictors of IGT performance. Similarly, Crone, Vendel and van der Molen (2003) compared two groups of adolescents (aged 12-13 years and 15-16 years) with young adults (18-25 years) and found that optimal IGT performance increased with age. Given 
the IGT resembles real-life decisions in terms of reward, punishment, and uncertainty of outcomes, researchers have argued that performance in this task involves the ventromedial prefrontal cortex, that is activated when individuals make choices that imply uncertain rewards and punishments (Critchley et al., 2001; Elliott et al., 2000; Ernst et al., 2002). A recent study demonstrated that decision-making improves in a J-shaped curve, with younger participants (aged 8-9 years) performing less disadvantageously than early adolescents (aged 12-13 years), because of a lesser sensitivity to rewards, and older adolescents making the most advantageous choices, because of the acquired ability to inhibit impulses, due to more mature development of the prefrontal cortex (Smith et al., 2012).

In summary, the psychological literature has shown that adults perform better than adolescents on the IGT, who, in turn, perform better than children as demonstrated by (i) the positive relationship between age and IGT performance (Crone \& van der Molen, 2004; Crone et al., 2005; Hooper et al., 2004), and (ii) ascribed poor decision-making to an non-linear development of subcortical and prefrontal regions that underpin decisionmaking (Smith et al., 2012). This decision-making impairment, exemplified by the perseverance of bad choices when performing the IGT, has been detected in several studies utilizing psychopathological populations, such as drug- and alcohol-addicted (Bechara et al., 2001; Goudriaan et al., 2005, 2006; Lorains et al., 2014; Monterosso et al., 2001; Wiehler \& Peters, 2015). Nevertheless, one question left unanswered by previous research is whether poor decision-making is associated with severity of gambling problems in adolescent gamblers (as has been found among adult gamblers). Comparing decision-making performance in adolescents with and without gambling problems is necessary to determine if poor decision-making abilities contribute to youth gambling problems. 
In addition to decision-making impairment, problem gambling is characterized by irrational biases that distort probabilistic predictions of wins and losses and lead individuals to hold an unjustified confidence in their ability to recover losses, and that can result in chasing losses. Even in chance-determined gambling, such as playing slot machines or roulette, gamblers believe they are able to influence the outcome of the game, through strategies or superstitious gestures that associate unrelated events (Toneatto et al., 1997). A large body of research has supported the role of cognitive distortions in gambling, demonstrating that over $70 \%$ of gambling-related cognitions externalized during gambling sessions are irrational (Griffiths, 1995; Ladouceur et al., 1991).

In research investigating adolescent gambling, Delfabbro and colleagues (2006) assessed adolescents' knowledge about the role of randomness, personal abilities, and the objective odds in gambling. They found that problem gamblers, despite having a good understanding of probabilities, were more confident in the possibility of influencing chance-determined events. More recently, cognitive distortions have been found to be strong predictor of youth gambling problems (Cosenza et al., 2014; Cosenza \& Nigro, 2015).

Problem gambling has also been associated with multiple psychiatric conditions, including substance and alcohol abuse (Blanco et al., 2001). The frequent co-occurrence of addiction disorders is not just restricted to adult populations. Adolescent gambling is typically associated with several risktaking behaviors, including substance and alcohol abuse (Vitaro et al., 2001; Winters et al., 1993). Moreover, the comorbidity appears to exacerbate gambling severity. For instance, Duhig and colleagues (2007) showed that adolescent frequent drinkers were more likely to report higher levels of engagement in gambling, when compared with non-drinkers peers. Liu and colleagues (2009) reported that substance-abusing adolescent gamblers began gambling at an earlier age and reported more problematic gambling 
involvement, when compared to non-substance-abusing adolescent gamblers. Given the common shared etiology and symptomatology, the involvement in one addiction may increase the likelihood of involvement in another.

Given this background, the present study attempted to expand our understanding of the development of gambling problems in adolescents. The first aim of the present study was to determine if adolescent decisionmaking is affected by level of gambling involvement. A second aim was to examine cognitive distortions and alcohol use in the presence and in the absence of gambling problems. A third aim was to examine the pattern of associations between gambling severity, decision-making, cognitive distortions, and alcohol use, and to identify the best predictors of problem gambling.

As has been found in adult problem gamblers, it was hypothesized that compared to adolescent non-problem gamblers, adolescent problem gamblers would have a poorer decision-making ability, higher cognitive distortions, and greater alcohol problems. It was also hypothesized that there would be significant associations between problem gambling, cognitive distortions, alcohol use, and poor decision-making.

\section{Method}

\section{Participants}

The sample comprised 104 adolescents males aged 16 to 19 years $\left(\mathrm{M}_{\mathrm{age}}=17.75\right.$ years; $\left.\mathrm{SD}_{\mathrm{age}}=0.84\right)$ attending the third, fourth and the fifth year of a secondary school situated in the province of Naples. Participants were randomly selected from a large sample of adolescents, based on SOGS-RA scores (South Oaks Gambling Screen Revised for Adolescents, Winters et al., 1993). Only male adolescents participated in this study because there were too few female problem gamblers. Previous studies (e.g., Blanco, 
Hasin, Petry, Stinson, \& Grant, 2006) found females start gambling later than males. Consequently, it is likely that the age group under investigation (16-19 years) does not include many female problem gamblers. The sample of non-problem gamblers (NPGs) comprised adolescents with a SOGS-RA score of 0 or 1 , whereas the sample of problem gamblers (PGs) ${ }^{1}$ comprised adolescents with a SOGS-RA of 4 or more. The two groups were matched for age $(\mathrm{F}(1,102)=2.68, p=.10)$ and class year.

\section{Measures}

Iowa Gambling Task (IGT; Bechara et al., 1994). The IGT is a computerized task that assesses decision-making abilities, and in which participants have to select one card at time from four decks of cards (A, B, C, D). Unbeknownst to the participant, two decks (A, B) give high immediate rewards, but are disadvantageous in the long-term because they result in high losses, whereas the other two decks (C, D) give lower immediate rewards, but are advantageous in the long run because they result in few losses. During the course of the task, participants learn the best strategy to maximize their imaginary final profits. Performance on the IGT is computed by subtracting the number of disadvantageous choices (A, B) from the number of advantageous choices $(C, D)$. The same computation for each block of 20 cards allows investigators to evaluate the changes in decision-making strategies across the task. A global score below 10 (out of 100 ) is indicative of a decision-making deficit (Bechara \& Damasio, 2002).

South Oaks Gambling Screen Revised for Adolescents (SOGS-RA; Winters et al., 1993; Italian SOGS-RA validation by Chiesi et al., 2013). The SOGSRA is a variant of the SOGS and evaluates the severity of gambling problems among adolescents. It comprises 16 items with dichotomous

\footnotetext{
1 In the DSM-IV (American Psychiatric Association, 1994), excessive problematic gambling behaviour was termed "pathological gambling", whereas in the DSM-5 (American Psychiatric Association, 2013), the name was changed to "gambling disorder"' (Petry et al., 2014)".
} 
(yes/no) answers, related to gambling behavior over the past year. A score between 0 and 1 denotes "non-problem" gambling, a score between 2 and 3 denotes "at-risk" gambling, and a score of 4 or more denotes "problem" gambling. In the present study, Cronbach's alpha (CA) was adequate at .76 $(95 \%$ CI $[.69, .82])$.

Gambling Related Cognitions Scale (GRCS; Raylu \& Oei, 2004; Italian validation by Iliceto et al., 2015). The GRCS assesses five dimensions of gambling-related irrational beliefs: gambling expectancies are expected benefits (as mood improvement) from gambling; illusion of control is the perceived ability to control gambling outcomes; predictive control is the misattribution of cause-and-effect relationships to unlinked events; inability to stop is the perceived inability to stop gambling behavior; and interpretative bias is an error of assessment, such as attributing wins to personal abilities. The GRCS comprises 23 items with 7-point Likert scale answers. Higher scores indicate higher levels of irrational belief. In the present study, CA for the total scale $(\alpha=.90,95 \%$ CI $[.87, .93])$ and for each scale was adequate: gambling-related expectancies $(\alpha=.68,95 \% \mathrm{CI}$ $[.57, .77])$, illusion of control ( $\alpha=.57,95 \%$ CI $[.42, .69])$, predictive control $(\alpha=.69,95 \%$ CI $[.59, .78])$, perceived inability to stop $(\alpha=.84,95 \%$ CI $[.79, .88])$, and interpretative bias $(\alpha=.82,95 \%$ CI $[.75, .87])$.

Alcohol Use Disorders Identification Test (AUDIT; Saunders et al., 1993; translated into Italian for the present study). The AUDIT is a 10-item selfreport screening measure comprising three questions concerning the amount and frequency of drinking alcohol, three questions concerning alcohol dependence, and four questions concerning problems caused by drinking alcohol. Each question is scored from 0 (never) to 4 (daily or almost daily) and a score of 8 or more indicates a strong likelihood of hazardous or harmful alcohol consumption. In the present study, CA for the AUDIT was .84 (95\% CI $[.78, .88])$. 


\section{Procedure}

Informed consent from the head of the school was obtained prior to data collection, following a letter clarifying the objectives of the research and the tools that would be used. After this, informed consent was obtained from students or their parents if they were underage (under 18 years). Participants were informed that they would be participating in an exploratory study on gambling, that the study would be anonymous, and that they could withdraw at any time. Data collection was held in two phases. In the first phase, participants completed a battery of paper-and-pencil questionnaires in the classroom, for a total duration of 30 minutes. In the second phase, each participant performed the computerized experimental task (i.e., the IGT), individually, in a school classroom. After the experimental session, participants were debriefed.

\section{Statistical analyses}

Analyses were performed with the Statistical Package for the Social Sciences (IBM SPSS Statistics for Windows, Version 20.0). The alpha significance level was set at .05. A univariate analysis of variance (ANOVA) with alcohol use total score (AUDIT) as the dependent variable and Group as independent variable (non-problem gamblers vs. problem gamblers) was performed to verify whether this measure differed significantly between groups. A mixed 2 x 5 ANCOVA was run with Group (non-problem gamblers vs. problem gamblers) as the between-participants factor, IGT stages (5 blocks of 20 trials) as the within-factor, and AUDIT score as covariate. These calculations were performed with the purpose of assessing decision-making ability between groups and of evaluating if this ability is independent of the alcohol use. A univariate analysis of covariance (ANCOVA) was performed with cognitive distortions total score (GRCS) as the dependent variable, Group as independent variable (non-problem gamblers vs. problem gamblers) and AUDIT score as covariate to verify whether these measures differed significantly between groups, 
independently from alcohol use. A multivariate analysis of covariance (MANCOVA) on the subscales of GRCS with Group (non-problem gamblers vs. problem gamblers) as independent factor and AUDIT score as covariate was also performed. Zero-order correlations were run to test associations among measures. To investigate the predictive value of measures on severity of gambling problems, a logistic regression analysis with Group (non-problem gamblers vs. problem gamblers) as independent and IGT, GRCS and AUDIT total scores as dependent variables was performed.

\section{Results}

The level of alcohol use (AUDIT) was different between the two groups: PGs reported higher levels of problem drinking than NPGs (see Table 1). Analysis conducted on the IGT performance across the five stages demonstrated a main effect of Stage, $\mathrm{F}(4,98)=5.83, p<.001, \eta^{2}=0.19$. This indicated an improvement in the performance for all the participants during the course of the task. There was also a main effect of Group, $F(1,101)=$ 5.56, $p<.02, \eta^{2}=0.05$, indicating that NPGs performed better than PGs. No Group x Stage interaction was found, $\mathrm{F}(4,98)=0.57, p=.69$ (see Figure 1). Alcohol use was not found to have a significant effect, $\mathrm{F}(4,98)=1.69, p=.16$.

The PGs scored significantly higher than NPGs on irrational beliefs total score (GRCS), and on all its subscales, namely gambling expectancies, illusion of control, predictive control, inability to stop, and interpretative bias, with a significant effect of AUDIT score, $\mathrm{F}(1,101)=15.55, p<.001, \eta^{2}=$ 0.13. From correlational analysis it emerged that cognitive distortions (GRCS), poor decision-making (IGT) and problematic alcohol consumption (AUDIT) were all associated with problem gambling (SOGS-RA). Significant associations between cognitive distortions and alcohol use were observed (see Table 2), and decision-making (IGT) correlated with a 
specific dimension of cognitive distortions (i.e., interpretative bias) (see Table 2).

Logistic regression was computed, entering the behavioral and self-report measures as predictors, and PG severity as criterion. In the first step, cognitive distortions (GRCS) was a significant predictor of gambling disorder, $\chi^{2}(1, n=104)=43.92, p<.001$. The percentage of explained variance with cognitive distortions as the only predictor was $46 \%$ (Nagelkerke $\mathrm{R}^{2}$ ). In the second step, along with cognitive distortions, alcohol consumption (AUDIT) was entered in the regression model and was significant, $\chi^{2}(2$, $\mathrm{n}=104)=4.59, p<.05$, and added $4 \%$ of the explained variance to the model. Finally, poor decision-making (IGT) was entered in the regression model and was significant, $\chi^{2}(3, n=104)=4.22, p<.05$, adding the $3 \%$ of the explained variance to the model. Consequently, the final regression model explained $53 \%$ of the variance of the criterion, with an overall classification accuracy of $78.8 \%$, and showed that cognitive distortions, decision-making, and alcohol consumption resulted in a higher likelihood to develop problem gambling (see Table 3).

\section{Discussion}

The present study is the first to examine the role of decision-making, cognitive distortions, and alcohol consumption in a sample of adolescents with and without gambling problems. One of the purposes of the study was to examine whether adolescent problem gamblers display the same psychological and behavioral patterns that have been identified in previous psychological literature examining adult problem gamblers.

The results clearly demonstrated that adolescent problem gamblers were impaired in decision-making, reported more problematic alcohol consumption, and had more gambling-related irrational beliefs, compared to non-problem gamblers peers. Significant associations between gambling 
severity, cognitive distortions and alcohol problems were observed. Poor decision-making (using IGT) was associated with a specific cognitive distortion (i.e., interpretative bias). Regression analyses confirmed that cognitive distortions, alcohol use, and poor decision-making were powerful predictors of problem gambling severity in adolescents.

The impaired decision-making performance found in adolescent problem gamblers (compared to non-problem gamblers) as indicated by frequent selections of cards from the disadvantageous decks. This result was largely consistent with many previous findings that have shown diminished performance on the IGT in problem gamblers (Forbush et al., 2008; Lakey et al., 2007). Poor decision-making has been ascribed to an 'insensitivity to future consequences' that concerns the difficulty to learn from previous mistakes and the inability to waive immediate and conspicuous rewards in favour of less consistent rewards that maximize profits in the long-term. Bechara and colleagues (1998) hypothesized that at the basis of this 'myopia for the future' there is an inability to use emotional processes in making choices. These emotional processes, which occur as somatic states, predict the advantages and disadvantages of each option, helping individuals in the choice. Similarly to prefrontal cortex patients (Damasio, 1994), problem gamblers were unable to use these somatic states to make good decisions. Given the frequent comorbidity between substance addiction and behavioral addiction (e.g., Liu et al., 2009), in the present study, the decision-making impairment observed in participants with an excessive gambling was controlled for in relation to alcohol use.

The negative correlation between problem gambling and decision-making suggests that the more severe the involvement, the more impaired the decision-making is. Interestingly, an association between decision-making deficit and a specific dimension of irrational beliefs (i.e., interpretative bias) was found. Interpretative bias specifically relates to the tendency to relate 
wins to personal skills and losses to bad luck. This association indicates that erroneous cognitions about gambling outcomes and the beliefs about being able to influence winning may worsen decision-making in gambling.

In the present study, youth problem gamblers reported more irrational beliefs respect to non-problem gamblers. In particular, they had irrational expectancies from gambling, such as receiving social approval from the game, and believing in their ability to influence and predict the outcome of the gamble. Moreover, they think they are unable to stop gambling, and tend to attribute wins to personal disposition and losses to situational factors. Previous studies have found that youth problem gamblers, even if they have an intact knowledge of mathematical rules and probability, tend to rely on rapid and economic reasoning that lead to systematic errors of assessment (Cosenza et al., 2014; Cosenza \& Nigro, 2015; Delfabbro et al., 2006). Although there is a large body of research concerning the role of cognitive distortions in the adult problem gamblers (e.g., Goodie \& Fortune, 2013; Griffiths, 1994; Joukhador et al., 2004; Raylu \& Oei, 2004), few studies have been conducted on adolescents. The present study, alongside previous research, confirms that cognitive distortions appear to be an important factor in the onset and development of problem gambling and that they predict the excessive gambling behavior. Furthermore, it has been demonstrated that problematic alcohol use is associated with irrational cognitions and may exacerbate gambling involvement.

Higher problematic alcohol use found among youth problem gamblers in the present study and the correlation between alcohol- and gambling-related problems confirms previous research showing a higher likelihood of addiction comorbidity (e.g., cigarette, alcohol, and/or drug use) among adolescents with gambling problems (Duhig et al., 2007; Liu et al., 2009; Winters et al., 1993). This is ascribed to the fact that many risk factors, such as family history, low self-esteem, and depression (Stinchfield \& Winters, 
1998) are common to both disorders, and that the engagement in one addictive behavior may increase the likelihood of developing another. The association between gambling and alcohol problems found in the present study is in line with a longitudinal research showing that alcohol abuse is a predictor of problematic gambling involvement in youth males (Barnes et al., 2002).

Finally, the present study demonstrated that decision-making deficits, cognitive distortions, and problematic alcohol consumption were the strong predictors of problem gambling in adolescents. It is also the first to demonstrate using an experimental and self-report paradigm that adolescent and adult problem gamblers have the same psychological and behavioral profile, characterized by irrational beliefs related to gambling outcomes, high willingness to be involved in other potential addicted behaviors (e.g., alcohol use), and decision-making impairment.

Previous research on decision-making in adolescent gamblers has not considered the associations with problem gambling using experimental methods. Such literature on decision-making has ascribed poor adolescent decisional abilities as being due to incomplete maturational brain development at this stage in the adolescent's life (Steinberg, 2005).

Although adequate, the relatively low internal reliability of the GRCS is one of the limitations of the present study. Another limitation of this study is its cross-sectional nature and the weaknesses that are inherent in both experimental and self-report methodologies. Further longitudinal studies that follow participants over the course of their adolescent development would be useful. Future brain-imaging studies could be used to examine decision-making impairments to confirm the results in the present study and to garner a more detailed understanding of the decisional patterns observed in youth problem gamblers. 
The findings of the present study demonstrated that gambling involvement appears negatively affect decision-making ability that is already deficient in adolescents. It is reasonable to assume that gambling activities hold more of an allure to adolescents (compared to adults), because of their still maturing brain development that creates a period of vulnerability for several behaviors that could result detrimental in the long-term, such as addictive behaviors. Furthermore, it is a vicious circle, because gambling experiences may worsen an individual's decision-making skills. This hypothesis explains why, given the same conditions of development, youth problem gamblers were more impaired in relation to their decision-making ability compared to non-problem gamblers peers. 


\section{Compliance with Ethical Standards}

Conflict of interest All other authors declare that they have no conflicts of interest.

Ethical Approval All procedures performed in studies involving human participants were in accordance with the ethical standards of the institutional and/or national research committee and with the 1964 Helsinki declaration and its later amendments or comparable ethical standards. 


\section{References}

American Psychiatric Association. (1994). Diagnostic and statistical manual of mental disorders (DSM). Washington, DC: American Psychiatric Association.

American Psychiatric Association (2013). Diagnostic and Statistical Manual of Mental Disorders (5th ed.). Arlington, VA: American Psychiatric Publishing.

Barnes, G. M., Welte, J. W., Hoffman, J. H., \& Dintcheff, B. A. (2002). Effects of alcohol misuse on gambling patterns in youth. Journal of Studies on Alcohol, 63(6), 767-775.

Bechara, A., \& Damasio, H. (2002). Decision-making and addiction (part I): impaired activation of somatic states in substance dependent individuals $\underline{\text { when pondering decisions with negative future }}$ consequences. Neuropsychologia, 40, 1675-1689.

Bechara, A., Damasio, A. R., Damasio, H., \& Anderson, S. W. (1994). Insensitivity to future consequences following damage to human prefrontal cortex. Cognition, 50, 7-15.

Bechara, A., Damasio, H., Tranel, D., \& Anderson, S. W. (1998). Dissociation of working memory from decision making within the human prefrontal cortex. Journal of Neuroscience, 18, 428-437.

Bechara, A., Dolan, S., Denburg, N., Hindes, A., Anderson, S., \& Nathan, P. (2001). Decision-making deficits, linked to a dysfunctional ventromedial prefrontal cortex, revealed in alcohol and stimulant abusers. Neuropsychologia, 39, 376-389.

Blanco, C., Hasin, D. S., Petry, N., Stinson, F. S., \& Grant, B. F. (2006). Sex differences in subclinical and DSM-IV pathological gambling: results from the National Epidemiologic Survey on Alcohol and Related Conditions. Psychological Medicine, 36, 943-953.

Blanco, C., Moreyra, P., Nunes, E. V., Saiz-Ruiz, J., \& Ibanez, A. (2001, July). Pathological gambling: addiction or compulsion? Seminars in Clinical Neuropsychiatry, 6(3), 167-176). 
Cassotti, M., Houdé, O., \& Moutier, S. (2011). Developmental changes of win-stay and loss-shift strategies in decision making. Child Neuropsychology, 17, 400-411.

Cauffman, E., Shulman, E. P., Steinberg, L., Claus, E., Banich, M. T., Graham, S., \& Woolard, J. (2010). Age differences in affective decision making as indexed by performance on the Iowa Gambling Task. Developmental Psychology, 46, 193-207.

Chiesi, F., Donati, M. A., Galli, S., \& Primi, C. (2013). The suitability of the South Oaks Gambling Screen-Revised for Adolescents (SOGS-RA) as a screening tool: IRT-based evidence. Psychology of Addictive Behaviors, 27, 287-293.

Cosenza, M., Baldassarre, I., Matarazzo, O., \& Nigro, G. (2014). Youth at stake: alexithymia, cognitive distortions, and problem gambling in late adolescents. Cognitive Computation, 6, 652-660.

Cosenza, M., \& Nigro, G. (2015). Wagering the future: Cognitive distortions, impulsivity, delay discounting, and time perspective in adolescent gambling. Journal of Adolescence, 45, 56-66.

Critchley, H. D., Mathias, C. J., \& Dolan, R. J. (2001). Neural activity in the human brain relating to uncertainty and arousal during anticipation. Neuron, 29, 537-545.

Crone, E. A., Bunge, S. A., Latenstein, H., \& van der Molen, M. W. (2005). Characterization of children's decision making: sensitivity to punishment frequency, not task complexity. Child Neuropsychology, 11, 245-263.

Crone, E. A., \& van der Molen, M. W. (2004). Developmental changes in real life decision making: performance on a gambling task previously shown to depend on the ventromedial prefrontal cortex. Developmental Neuropsychology, 25(3), 251-279.

Crone, E. A., Vendel, I., \& van der Molen, M. W. (2003). Decision-making in disinhibited adolescents and adults: insensitivity to future consequences or driven by immediate reward? Personality and Individual Differences, 35, 1625-1641. 
Damasio, A. R. (1994). Descartes' error: Emotion, rationality and the human brain. New York: Avon Books.

Delfabbro, P., Lahn, J., \& Grabosky, P. (2006). It's not what you know, but how you use it: Statistical knowledge and adolescent problem gambling. Journal of Gambling Studies, 22, 179-193.

Duhig, A. M., Maciejewski, P. K., Desai, R. A., Krishnan-Sarin, S., \& Potenza, M. N. (2007). Characteristics of adolescent past-year gamblers and non-gamblers in relation to alcohol drinking. Addictive Behaviors, 32(1), 80-89.

Elliott, R., Dolan, R. J., \& Frith, C. D. (2000). Dissociable functions in the medial and lateral orbitofrontal cortex: Evidence from human neuroimaging studies. Cerebral Cortex, 10, 308-317.

Ernst, M., Bolla, K., Mouratidis, M., Contoreggi, C., Matochik, J. A., Kurian, V., et al. (2002). Decision-making in a risk-taking task: A PET study. Neuropsychopharmacology, 26, 682-691.

Forbush, K. T., Shaw, M., Graeber, M. A., Hovick, L., Meyer, V. J., Moser, D. J., ... \& Black, D. W. (2008). Neuropsychological characteristics and personality traits in pathological gambling. CNS Spectrums, 13, 306-315.

Goodie, A. S., \& Fortune, E. E. (2013). Measuring cognitive distortions in pathological gambling: Review and meta-analyses. Psychology of Addictive Behaviors, 27(3), 730-743.

Goudriaan, A. E., Oosterlaan, J., de Beurs, E., \& van den Brink, W. (2005). Decision making in pathological gambling: a comparison between pathological gamblers, alcohol dependents, persons with Tourette syndrome, and normal controls. Cognitive Brain Research, 23, 137-151.

Goudriaan, A. E., Oosterlaan, J., de Beurs, E., \& van den Brink, W. (2006). Psychophysiological determinants and concomitants of deficient decision making in pathological gamblers. Drug and Alcohol Dependence, 84, 231239.

Griffiths, M. D. (1994). The role of cognitive bias and skill in fruit machine gambling. British Journal of Psychology, 85(3), 351-369. 
Griffiths, M. D. (1995). Adolescent gambling. London: Routledge.

Hooper, C. J., Luciana, M., Conklin, H. M., \& Yarger, R. S. (2004). Adolescents' performance on the Iowa Gambling Task: implications for the development of decision making and ventromedial prefrontal cortex. Developmental Psychology, 40, 1148-1158.

Iliceto, P., Fino, E., Cammarota, C., Giovani, E., Petrucci, F., Desimoni, M., ... \& Oei, T. P. (2015). Factor structure and psychometric properties of the Italian version of the Gambling Related Cognitions Scale (GRCS-I). Journal of Gambling Studies, 31, 225-242.

Joukhador, J., Blaszczynski, A., \& Maccallum, F. (2004). Superstitious beliefs in gambling among problem and non-problem gamblers: Preliminary data. Journal of Gambling Studies, 20(2), 171-180.

Ladouceur, R., Gaboury, A., Bujold, A., Lachance, N., \& Tremblay, S. (1991). Ecological validity of laboratory studies of videopoker gaming. Journal of Gambling Studies, 7, 109-116.

Lakey, C. E., Goodie, A. S., \& Campbell, W. K. (2007). Frequent card playing and pathological gambling: The utility of the Georgia Gambling Task and Iowa Gambling Task for predicting pathology. Journal of Gambling Studies, 23, 285-297.

Liu, T., Maciejewski, P. K., \& Potenza, M. N. (2009). The relationship between recreational gambling and substance abuse/dependence: data from a nationally representative sample. Drug and Alcohol Dependence, 100, 164-168.

Lorains, F. K., Dowling, N. A., Enticott, P. G., Bradshaw, J. L., Trueblood, J. S., \& Stout, J. C. (2014). Strategic and non-strategic problem gamblers differ on decision-making under risk and ambiguity. Addiction, 109, 11281137.

Monterosso, J., Ehrman, R., Napier, K. L., O’Brien, C. P., \& Chidress, A. R. (2001). Three decision-making tasks in cocaine-dependent patients: Do they measure the same construct? Addiction, 96, 1825-1837. 
Petry, N. M., Blanco, C., Auriacombe, M., Borges, G., Bucholz, K., Crowley, et al. (2014). An overview of and rationale for changes proposed for pathological gambling in DSM-5. Journal of Gambling Studies, 30, 493502 .

Raylu, N., \& Oei, T. P. (2004). The Gambling Related Cognitions Scale (GRCS): Development, confirmatory factor validation and psychometric properties. Addiction, 99, 757-769.

Saunders, J. B., Aasland, O. G., Babor, T. F., De la Fuente, J. R., \& Grant, M. (1993). Development of the alcohol use disorders identification test (AUDIT). WHO collaborative project on early detection of persons with harmful alcohol consumption-II. Addiction, 88, 791-804.

Smith, D. G., Xiao, L., \& Bechara, A. (2012). Decision making in children and adolescents: impaired Iowa Gambling Task performance in early adolescence. Developmental Psychology, 48, 1180-1187.

Steinberg, L. (2005). Cognitive and affective development in adolescence. Trends in Cognitive Sciences, 9, 69-74.

Stinchfield, R. D., \& Winters, K. C. (1998). Adolescent gambling: A review of prevalence, risk factors and health implications. Annals of the American Academy of Political and Social Science, 556, 172-185.

Toneatto, T., Blitz-Miller, T., Calderwood, K., Dragonetti, R., \& Tsanos, A. (1997). Cognitive distortions in heavy gambling. Journal of Gambling Studies, 13, 253-266.

Vitaro, F., Brendgen, M., Ladouceur, R., \& Tremblay, R. E. (2001). Gambling, delinquency, and drug use during adolescence: Mutual influences and common risk factors. Journal of Gambling Studies, 17, 171190.

Wiehler, A., \& Peters, J. (2015). Reward-based decision making in pathological gambling: the roles of risk and delay. Neuroscience Research, 90, 3-14. 
Winters, K. C., Stinchfield, R. D., \& Fulkerson, J. (1993). Toward the development of an adolescent gambling problem severity scale. Journal of Gambling Studies, 9, 63-84. 
Table 1. Mean and standard deviations of the self-report measures for non-problem gamblers (NPGs) and problem gamblers (PGs).

\begin{tabular}{llllllll}
\hline \multicolumn{7}{l}{ NPGs $(\boldsymbol{N}=\mathbf{5 2})$} & \multicolumn{7}{l}{ PGs $(\mathbf{N = 5 2 )}$} & & & \\
\hline & M & SD & M & SD & $\boldsymbol{F ( 1 , 1 0 2 )}$ & $\boldsymbol{p}$ & $\boldsymbol{\eta}^{\mathbf{2}}$ \\
GRCS-GE & 7.58 & 3.76 & 10.61 & 4.34 & 14.46 & .001 & .12 \\
GRCS-IC & 5.58 & 2.67 & 7.85 & 3.73 & 12.74 & .01 & .11 \\
GRCS-PC & 12.63 & 6.00 & 17.65 & 6.08 & 17.94 & .001 & .15 \\
GRCS-IS & 7.08 & 2.33 & 14.77 & 6.57 & 63.25 & .001 & .38 \\
GRCS-IB & 8.17 & 4.70 & 14.96 & 4.92 & 51.71 & .001 & .34 \\
GRCS-TOT & 41.04 & 14.66 & 65.85 & 18.70 & 56.66 & .001 & .36 \\
AUDIT & 3.61 & 3.26 & 8.52 & 6.50 & 23.64 & .001 & .19 \\
IGT & 29.54 & 19.90 & 17.81 & 25.60 & 6.80 & .05 & .06 \\
\hline
\end{tabular}

Note. GRCS-GE $=$ Gambling Expectances scale of GRCS; GRCS-IC $=$ Illusion of Control scale of GRCS; GRCS-PC = Predictive Control scale of GRCS; GRCS-IS = Inability to Stop scale of GRCS; GRCS-IB = Interpretative Bias scale of GRCS; GRCS-TOT = Gambling Related Cognition Scale Total Score; AUDIT = Alcohol Use Disorders Identification Test; IGT = Iowa Gambling Task. 
Table 2. Pearson correlation coefficients among measures among non-problem gamblers $(\mathrm{n}=52)$ and problem gamblers $(\mathrm{n}=52)$

\begin{tabular}{lllllllll}
\hline & 1 & 2 & 3 & 4 & 5 & 6 & 7 & 8 \\
\hline 1.SOGS-RA & - & & & & & & \\
2.GRCS-GE & $.40 * *$ & - & & & & & \\
3.GRCS-IC & $.42 * *$ & $.39 * *$ & - & & & & & \\
4.GRCS-PC & $.42 * *$ & $.53 * *$ & $.54 * *$ & - & & & & \\
5.GRCS-IS & $.66 * *$ & $.58 * *$ & $.37 * *$ & $.47 * *$ & - & & & \\
6.GRCS-IB & $.63 * *$ & $.48 * *$ & $.42 * *$ & $.67 * *$ & $.61 * *$ & - & & \\
7.GRCS-TOT & $.66 * * * *$ & $.75 * * *$ & $.64 * *$ & $.84 * *$ & $.80 * *$ & $.84 * *$ & - & \\
8.AUDIT & $.53 * *$ & $.38 * *$ & $.40 * *$ & $.39 * *$ & $.53 * *$ & $.37 * *$ & $.52 * *$ & - \\
9.IGT &. $.30 * *$ & .02 & .02 & -.13 & -.11 & $-.27 * *$ & -.14 & -.10 \\
\hline
\end{tabular}

Bold values represent significant correlation coefficients.

Note. SOGS-RA = South Oaks Gambling Screen Revised for Adolescents; GRCS-GE = Gambling Expectances scale of GRCS; GRCS-IC = Illusion of Control scale of GRCS; GRCS-PC = Predictive Control scale of GRCS; GRCS-IS = Inability to Stop scale of GRCS; GRCS-IB = Interpretative Bias scale of GRCS; GRCS-TOT = Gambling Related Cognition Scale Total Score; AUDIT = Alcohol Use Disorders Identification Test; IGT = Iowa Gambling Task.

$* p<.05, * * p<.01$ 
Table 3. Multivariate prediction of severity of problem gambling using linear regression analysis

\begin{tabular}{lllll}
\hline Predictors & Beta & S.E. & Wald & value \\
& & & Statistic & \\
\hline Step 1 & & & 0.000 \\
GRCS-TOT & 0.08 & 0.02 & 26.73 & \\
\hline Step 2 & & & 0.000 \\
GRCS-TOT & 0.07 & 0.02 & 19.03 & 0.04 \\
AUDIT & 0.13 & 0.06 & 4.11 & \\
\hline Step 3 & & & 0.000 \\
GRCS-TOT & 0.07 & 0.02 & 17.58 & 0.05 \\
IGT & -0.02 & 0.01 & 3.67 & 0.04 \\
AUDIT & 0.13 & 0.06 & 4.16 & \\
\hline
\end{tabular}

Note. GRCS-TOT = Gambling Related Cognition Scale Total Score; AUDIT $=$ Alcohol Use Disorders Identification Test; IGT = Iowa Gambling Task. 


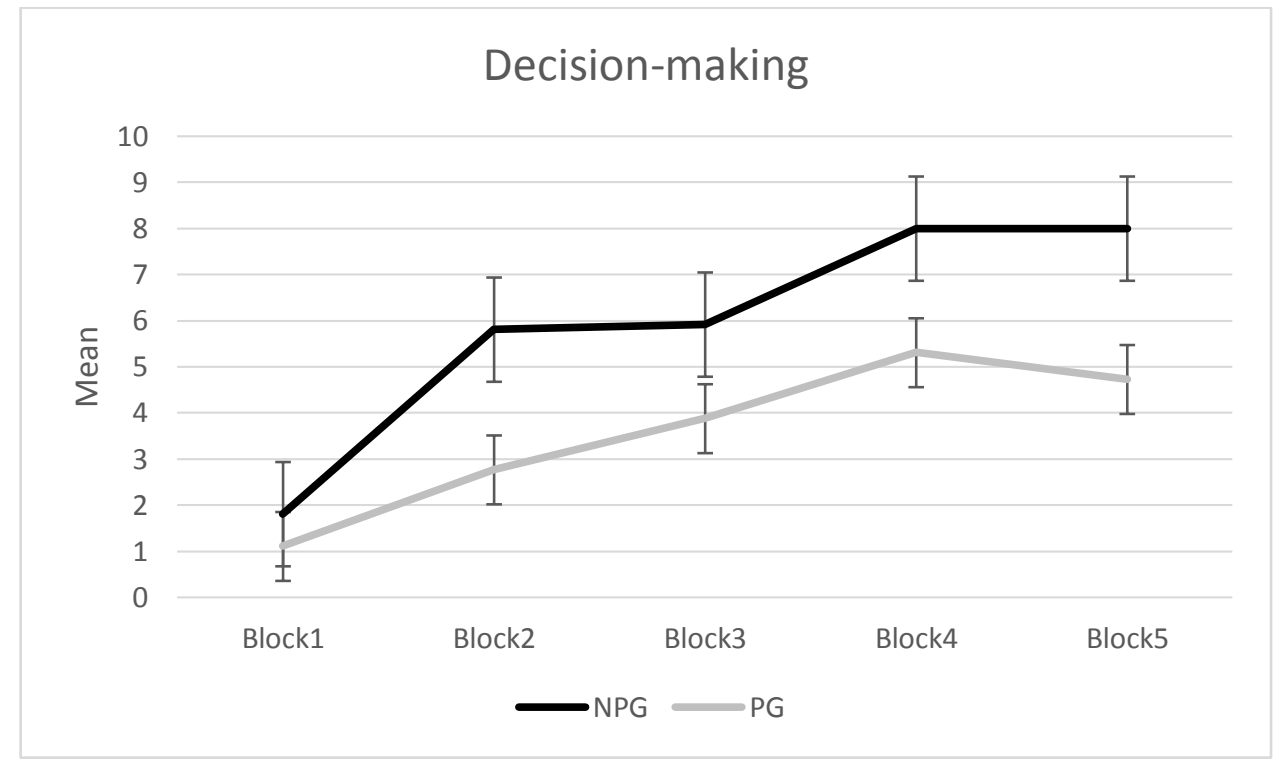

Figure 1. Means and the standard errors of the mean (SEM) of cards chosen from advantageous minus disadvantageous decks over the five IGT blocks as function of Group. 